\title{
From Electroclinical to Electrometabolic Status Epilepticus?
}

\author{
Gregory Kapinos $^{1} \cdot$ Jan Claassen $^{2}$
}

Published online: 12 May 2016

(c) Springer Science+Business Media New York 2016

Electroencephalographic periodic discharges (PDs) have a complex clinical significance and carry unclear therapeutic implication [1-7], best understood along the "ictal-interictal continuum" (IIC) put forth by Chong and Hirsch [3]. These epileptiform patterns are not a homogenous group, with diverse electroencephalographic characteristics $[2,3$, $6,7]$, differing syndromal context [2,6], variable clinical expression [2-6], and most importantly, may have vastly different underlying etiologies and prognoses [1-6].

In order to define at what point PDs behave like nonconvulsive seizures (NCSZs), epilepsy and neurocritical care experts [1-7] proposed that there might be more of a gray zone along this IIC [3], than a clear dichotomy, ictal versus non-ictal [7]. Over the past decade, a debate has been ongoing about the clinical significance of these ambiguous "semi-ictal" patterns, also known as "boundary syndromes" $[2,6]$. As experts expressed their opinions on which patterns are harmful enough to warrant treatment $[1$, 3-5], a second axis has been proposed, where "injuriousness" or "worth treating" is separately represented on the $y$-axis, while keeping "ictal signature" on the $\mathrm{x}$-axis [3].

One avenue of research has identified a strong correlation between PDs on the IIC and subsequent NCSZs or non-convulsive status epilepticus (NCSE), with reviews articulating this gradation in severity of PDs [2, 3, 6, 7].

Gregory Kapinos

kapigreg@gmail.com

1 Departments of Neurosurgery and Neurology, Hofstra Northwell School of Medicine, 300 Community Drive, Tower, 9th Floor, Manhasset, NY 11030, USA

2 Department of Neurology, College of Physicians \& Surgeons, Columbia University, New York, NY, USA
Distinctively, another avenue of research identified which patterns are responsible for an altered brain function (from encephalopathy to coma), nicely compiled in recent synopses $[2,5,6]$, but the link between PDs on the IIC and functional outcome remains less certain [1-6]. Another direction of research studied the physiologic repercussions of PDs, ultimately suggesting a link between interictal periodic patterns and secondary brain injury (as inferred by increased vasogenic or cytotoxic edema $[8,9]$ or increase in lactate-pyruvate ratio [10]), seen as similar sequelae to those resulting from NCSZs [10-12]. Many questions remain, as causality has not been proven [1, 12], but inferences may be made from these physiologic characteristics [4] that have been evoked to guide management [4, 5].

This body of evidence led to the concept of a nosologic umbrella under which a patient, with EEG evidence of IIC that does not meet formal seizure criteria, would be considered to be in "status epilepticus" (SE), if the clinical exam and overall context are compelling for high risk of NCSE [2, 3, 5, 6, 13]. "Electro-clinical status epilepticus" (ECSE) is elegantly proposed as a term for this nosologic concept, by Struck et al. [13] in this issue of Neurocritical Care, defining what others have reported as "boundary syndromes" [2], "coma-NCSE" [2, 6], "coma-PDs" [6], or "harmful patterns on the IIC" [3-5].

In an effort to confirm the ictal, sedating/altering, or harmful nature of these IIC patterns, neurointensivists have advocated for the use of a benzodiazepine trial to test for ECSE $[4,5]$, but in brain-injured critically ill patients, this diagnostic study is often of limited practical value $[4,5]$. As an alternative approach, imaging studies have attempted to build the argument for the ictal nature of an IIC pattern in question, by demonstrating similarities of physiologic 
properties, such as blood flow [14, 15] or metabolic activation [16], to those of patients with definite seizures. A dozen studies characterize PDs with perfusion imaging, mainly using single photon emission computed tomography (SPECT) [14, 15], but distinctively, metabolic imaging has seldom scrutinized PDs, aside a large body of evidence for hypermetabolism seen for clear-cut seizures, notably in epilepsia partialis continua. One case series [17] and one case report [18] have documented co-localized areas of hypermetabolism with the regional foci of PDs, using positron emission tomography (PET).

To further clarify which PDs on the IIC are worthy of a vigorous anti-seizure regimen, Struck et al. [13], in this issue of Neurocritical Care, studied if hypermetabolism seen on PET was linked to subsequent seizures. In this article, the authors investigated 18-fluorodeoxyglucose (FDG)-PET imaging as a complementary metabolic surrogate of SE among patients with diverse IIC patterns, using a single-center prospectively collected case series [13]. Eighteen hospitalized subjects underwent FDG-PET during a time that EEG demonstrated IIC patterns. Hypermetabolism was found to be predictive of electrographic SE, as well as ECSE, and the latter independently from the former [13].

This paper is of great interest to any practicing neurointensivist using EEG with some regularity, and who faces these EEG periodic patterns of unclear significance on a daily basis, as it shifts the interest from cerebral blood flow to metabolism. This shift may provide a crucial piece of the unresolved puzzle of the IIC $[4,5]$. The data are preliminary, as clinical responses were determined on retrospective chart review and the treating practitioners were not been blinded to PET findings. Future studies may also look into later in-hospital seizures, later electrographic SE, as well as secondary seizure-induced lesions [8, 9, 11, 12], oxidative disarray [10], inflammation [19], and cognitive and functional outcomes [20].

The authors [13] only had data from a rather etiologically diverse patient cohort. This needs to be taken into account when interpreting the findings, as different etiologies may in themselves result in highly variable metabolic signatures. The data do not allow to convincingly unravel the chicken and egg problem, between controversial EEG patterns and brain injury [1, 12]. Also, as the timing of PET in relation to the onset of IIC was not standardized, some IIC patterns could have "burned out" before the metabolic imaging. The authors elegantly investigated hypermetabolism and hypometabolism, and further sub-classified hypermetabolism into focal, regional, or diffuse [13]. Larger studies that are powered to investigate these subgroups and analyze quantitative rather than qualitative metabolic changes associated with specific patterns, may be of interest.
Interestingly, the study [13] showed a trend for hypermetabolism correlating with IIC patterns that also demonstrated features that may be considered more "seizure-like," such as high-amplitude near-rhythmic PDs occurring for a longer time. Hypermetabolism may have some potential to allow distinguishing between "seizurelike" IIC patterns and "non-seizure-like" IIC patterns. If confirmed and also associated with measures of true injury or poor outcome, hypermetabolism may, in combination with EEG features, emerge as a biomarker of ECSE. In their Fig. 2, the authors [13] integrated hypermetabolism into Chong and Hirsch's continuum [3]. However, putting graded hypermetabolism in the top right corner of the continuum may send the message that metabolic imaging perfectly superimposes with the IIC of Chong and Hirsch. One may thus argue that metabolic characterization would not add significant independent information to the continuum and electroencephalographic characterization of the IIC might suffice, without any of this cumbersome metabolic neuroimaging.

For the practicing neurointensivist facing therapeutic decision, the clinical context, including underlying etiology, syndrome, and the presence of structural or inflammatory lesions, are each and all tightly linked to the risk of subsequent seizures and poor functional outcome. Metabolic imaging may aver to be a useful tool to better characterize these EEG periodic patterns of uncertain significance, but needs further study.

\section{Compliance with Ethical Standards}

Disclosures Dr. Kapinos reports 2014 honoraria from Clearview/ Medsource, related to consultation in study design for the SAGE-547 compound in super-refractory status epilepticus and from BARD for advisory consultation on critical care catheter design. Dr. Claassen is on the advisory board for study planning for SAGE Therapeutics, Actelion and BARD and is the site principal investigator for several $\mathrm{NIH}$-sponsored clinical trials.

\section{References}

1. Young GB, Claassen J. Nonconvulsive status epilepticus and brain damage: further evidence, more questions. Neurology. 2010;75(9):760-1.

2. Sutter R, Kaplan PW. Electroencephalographic criteria for nonconvulsive status epilepticus: synopsis and comprehensive survey. Epilepsia. 2012;53(Suppl 3):1-51.

3. Chong DJ, Hirsch LJ. Which EEG patterns warrant treatment in the critically ill? Reviewing the evidence for treatment of periodic epileptiform discharges and related patterns. J Clin Neurophysiol. 2005;22(2):79-91.

4. Claassen J. How I treat patients with EEG patterns on the ictalinterictal continuum in the neuro ICU. Neurocrit Care. 2009;11(3):437-44.

5. Sivaraju A, Gilmore EJ. Understanding and managing the ictalinterictal continuum in neurocritical care. Curr Treat Options Neurol. 2016;18(2):8. 
6. Bauer G, Trinka E. Nonconvulsive status epilepticus and coma. Epilepsia. 2010;51(2):177-90.

7. Beniczky S, Hirsch LJ, Kaplan PW, Pressler R, Bauer G, Aurlien $\mathrm{H}$, Brøgger JC, Trinka E. Unified EEG terminology and criteria for nonconvulsive status epilepticus. Epilepsia. 2013;54(Suppl 6):28-9.

8. Xiang T, Li G, Liang Y, Zhou J. A wide spectrum of variably periictal MRI abnormalities induced by a single or a cluster of seizures. J Neurol Sci. 2014;343(1-2):167-72.

9. Canas N, Breia P, Soares P, Saraiva P, Calado S, Jordão C, Vale $\mathrm{J}$. The electroclinical-imagiological spectrum and long-term outcome of transient periictal MRI abnormalities. Epilepsy Res. 2010;91(2-3):240-52.

10. Vespa P, Tubi M, Claassen J, Buitrago-Blanco M, McArthur D, Velazquez AG, Tu B, Prins M, Nuwer M. Metabolic crisis occurs with seizures and periodic discharges after brain trauma. Ann Neurol. 2016;79(4):579-90.

11. Vespa PM, O'Phelan K, Shah M, Mirabelli J, Starkman S, Kidwell C, Saver J, Nuwer MR, Frazee JG, McArthur DA, Martin NA. Acute seizures after intracerebral hemorrhage: a factor in progressive midline shift and outcome. Neurology. 2003;60(9): 1441-6.

12. Grillo E. Postictal MRI abnormalities and seizure-induced brain injury: notions to be challenged. Epilepsy Behav. 2015;44:195-9.

13. Struck AF, Westover B, Hall LT, Deck GM, Cole AJ, Rosenthal ES. Metabolic correlates of the ictal-interictal continuum: FDGPET during continuous EEG. Neurocrit Care. 2016 (In press).

14. Zeiler SR, Turtzo LC, Kaplan PW. SPECT-negative SIRPIDs argues against treatment as seizures. J Clin Neurophysiol. 2011;28(5):493-6.
15. Smith CC, Tatum WO, Gupta V, Pooley RA, Freeman WD. SPECT-negative SIRPIDs: less aggressive neurointensive care? J Clin Neurophysiol. 2014;31(3):e6-10.

16. Stefanovic B, Warnking JM, Kobayashi E, Bagshaw AP, Hawco C, Dubeau F, Gotman J, Pike GB. Hemodynamic and metabolic responses to activation, deactivation and epileptic discharges. Neuroimage. 2005;28(1):205-15.

17. Franck G, Sadzot B, Salmon E, Maquet P, Peter JM, Quaglia L, Delfiore G, Lamotte D. Study of cerebral metabolism and blood flow in partial complex epilepsy and status epilepticus in man using positron emission tomography. [Article in French]. Rev Electroencephalogr Neurophysiol Clin. 1986;16(3):199-216.

18. Handforth A, Cheng JT, Mandelkern MA, Treiman DM. Markedly increased mesiotemporal lobe metabolism in a case with PLEDs: further evidence that PLEDs are a manifestation of partial status epilepticus. Epilepsia. 1994;35(4):876-81.

19. Claassen J, Albers D, Schmidt JM, De Marchis GM, Pugin D, Falo CM, Mayer SA, Cremers S, Agarwal S, Elkind MS, Connolly ES, Dukic V, Hripcsak G, Badjatia N. Nonconvulsive seizures in subarachnoid hemorrhage link inflammation and outcome. Ann Neurol. 2014;75(5):771-81.

20. De Marchis GM, Pugin D, Meyers E, Velasquez A, Suwatcharangkoon S, Park S, Falo MC, Agarwal S, Mayer SA, Schmidt JM, Connolly ES, Claassen J. Seizure burden in subarachnoid hemorrhage associated with functional and cognitive outcome. Neurology. 2016;86(3):253-60. 